\title{
REPTILES EN EL MONUMENTO NATURAL EL MORADO (REGION METROPOLITANA, CHILE): ABUNDANCIA RELATIVA, DISTRIBUCION ALTITUDINAL Y PREFERENCIA POR ROCAS DE DISTINTO TAMAÑO
}

\author{
REPTILES FROM 'EL MORADO' NATURAL MONUMENT (REGION \\ METROPOLITANA, CHILE): RELATIVE ABUNDANCE, ALTITUDINAL \\ DISTRIBUTION, AND PREFERENCE BY DIFFERENT SIZES OF ROCKS
}

\author{
Jorge E. Mella \\ Escuela de Ecología y Paisaje, Universidad Central de Chile, Santiago. \\ Centro de Ecología Aplicada Ltda., Av. Suecia 3304, Ñuñoa, Santiago. Email: ima@cea.cl
}

\begin{abstract}
RESUMEN
En este estudio se caracterizó la fauna de reptiles presentes en el Monumento Natural El Morado, Región Metropolitana, Chile. Se encontraron seis especies (cuatro lagartijas y dos culebras), cuyas abundancias fueron: Liolaemus moradoensis $(40,9 \%)$, L. nigroviridis $(37,7 \%)$, L. valdesianus $(17,1 \%)$, L. nitidus $(2,7 \%)$, Tachymenis chilensis $(1,2 \%)$ y Philodryas chamissonis $(0,4 \%)$. A pesar del estrecho rango altitudinal estudiado (cerca de $600 \mathrm{~m}$ ), las distintas especies presentaron diferentes patrones de distribución: L. nitidus se encontró sólo en el piso altitudinal más bajo (menor a $1.900 \mathrm{msnm}$ ), mientras que L. moradoensis y L. valdesianus se encuentran sobre los $2.150 \mathrm{msnm}$ (alcanzando esta última especie los $2.450 \mathrm{msnm}$, con lo que se amplía el rango altitudinal conocido). La especie que ocupó un mayor rango de altitud fue L. nigroviridis, presente entre los 1.850 a los $2.350 \mathrm{msnm}$. En cuanto a la preferencia por rocas de distinto tamaño, L. moradoensis mostró preferencia por rocas entre 30 a $60 \mathrm{~cm}$. L. nigroviridis prefirió en alopatía rocas entre 30 a $120 \mathrm{~cm}$, y cambió su preferencia a rocas entre 30 a $60 \mathrm{~cm}$ cuando se encuentra en sintopía con L. valdesianus; esta última prefirió rocas mayores a $90 \mathrm{~cm}$. En términos de uso del nicho: para el eje del nicho tamaño de rocas, $L$. nigroviridis se sobrepone a $L$. moradoensis, especie que tiene un nicho más estrecho, mientras que $L$. valdesianus tiene un nicho no sobrepuesto, a mayor tamaño de rocas. Es interesante destacar un desplazamiento del nicho en L. nigroviridis, la única especie que se estudió en diferentes contextos de sintopía. Factores asociados a requerimientos termorregulatorios y probablemente la reducción de presiones competitivas podrían dar cuenta de los resultados observados.
\end{abstract}

Palabras claves: Reptiles saxicolas, Liolaemus, preferencia por rocas, nicho, Chile central.

\section{ABSTRACT}

This study caracterizes the reptilian fauna at El Morado Natural Monument in the Metropolitan Region of Chile. The relative abundance of lizards and snakes is described relative to altitude and the size of terrain boulders. Four species of lizards and two species of snakes were encountered; their observed relative abundances are: Liolaemus moradoensis (40,9\%), L. nigroviridis $(37,7 \%)$, L. valdesianus $(17,1 \%)$, L. nitidus $(2,7 \%)$, Tachymenis chilensis $(1,2 \%)$, and Philodryas chamissonis $(0,4 \%)$. The altitude range of the study area is 1,850 to 2,450 masl. L. nigroviridis was found throughout this altitudinal range. L. nitidus restricted to altitudes below 1,900 masl. L. moradoensis and L. valdesianus were observed no lower than 2,150 masl. (reaching L. valdesianus the 2,459 masl., increasing its previously known altitudinal range). With regard to boulder size preference, $L$. valdesianus demonstrated a preference for boulders with a long axis greater than $0.9 \mathrm{~m}$ in size. L. moradoensis preferred boulders 0.3 to $0.6 \mathrm{~m}$ in size. L. nigroviridis showed a preference for boulders $0.3-1.2 \mathrm{~m}$ in size below 2,150 masl, but above this altitude, where its distribution is syntopic with $L$. valdesianus, the distribution of $L$. nigroviridis was restricted to boulders in the 0.3-0.6 m size range. In terms of niche use (size of rocks as a niche axis): L. nigroviridis shows a niche superimposed to $L$. altissimus, who have a narrow niche, while L. valdesianus 
Reptiles en el Monumento Natural El Morado: Mella, J.

have a not superimposed niche, to greater size of rocks. It is interesting the displacement of niche in L. nigroviridis, the only species studied under different contexts of syntopy. Thermoregulatory factors and probably the reduction of competitive pressure could explain the patterns found.

Keywords: Reptiles saxicolous, Liolaemus, boulders, syntopic competition, niche, central Chile.

\section{INTRODUCCION}

El Monumento Natural (MN) El Morado, de 3.000 ha de extensión, se encuentra ubicado en la parte superior del río Volcán, en la Cordillera de los Andes, a 90 km. al SE de Santiago, en la Región Metropolitana de Chile. El rango de altitud de esta área protegida varía entre los 1.800 y los $5.060 \mathrm{msnm}$. El conocimiento de la biota del MN El Morado es escaso (CONAF 1988; Teillier et al. 1994, Teillier 2003), y en el caso de la flora, se han citado 300 especies de plantas vasculares, de las cuales 263 son nativas y 37 son exóticas (Teillier 2003) y al menos 45 son especies raras o de distribución restringida (Teillier et al. 1994). Para la fauna de vertebrados se menciona la presencia de 44 especies de aves, 3 anfibios, 3 reptiles y 5 mamíferos (CONAF 1988), y recientemente se ha estudiado la dieta de cernícalo y el tucúquere (Mella 2002), la dinámica poblacional de Bufo spinulosus (Mella 2006a), la abundancia relativa y cambios estacionales de los micromamíferos (Mella 2005b) y aves (Mella 2006c), pero se carece de antecedentes de la fauna de reptiles de la unidad.

Los reptiles presentes en áreas protegidas han sido escasamente estudiados (ej. Ortiz 1974; Jerez \& Ortiz 1975; Zunino \& Riveros 1981; Díaz \& Simonetti 1997; Moreno et al. 2000; Ortiz \& IbarraVidal 2005). Dentro de ellos, la distribución altitudinal de los reptiles ha sido estudiada sólo para el Parque Nacional La Campana (Jerez \& Ortiz 1975; Zunino \& Riveros 1981) y la Reserva Nacional Río Clarillo (Díaz \& Simonetti 1997).

La gran mayoría de las especies de lagartos y lagartijas de las zonas andinas de Chile central son saxícolas (Donoso-Barros, 1966), y aunque existen diversos estudios que han comparado el uso de diferentes microhábitats entre especies de lagartijas (ej. Fuentes \& Cancino, 1979; Jaksic \& Núñez 1979; Marquet et al. 1989; Núñez 1996; Carothers et al. 1998), dichos análisis comparan el uso de rocas con otros microhábitats, sin diferenciar entre distintas categorías de tamaño de rocas.
En términos de la teoría del nicho (Pianka 1973; Schoener 1974), el estudio de selección de rocas de distinto tamaño por lagartijas puede servir como ejemplo de selección y segregación de un eje particular (una de lasn dimensiones) del nicho, de modo de ser un mecanismo (vía división de recursos) para la evasión de la posible competencia interespecífica. Uno de los escasos estudios locales que analizan el uso de rocas de distinto tamaño es el de Carothers et al. (2001), quienes mencionan el uso de rocas grandes por Liolaemus leopardinus y de rocas pequeñas por L. monticola, aunque no presentan datos cuantitativos al respecto. Por otra parte, Fuentes \& Cancino (1979) mencionan la posible selección de microhábitat por L. kuhlmanni, especie presente siempre sobre o cerca de rocas grandes en la costa de la IV Región. Específicamente, no existen antecedentes cuantitativos para Liolaemus chilenos sobre la preferencia por rocas de distinto tamaño por las especies saxícolas, ni su análisis en términos de uso y cambio de nicho. Como factores explicativos del uso y/o preferencia de rocas por los reptiles se han mencionado aspectos termorregulatorios, evasión de competencia, preferencias para depredación y selección de pareja (Fuentes \& Cancino, 1979; Habit \& Ortiz 1994; Carothers et al. 1997, 1998, 2001; Vidal et al. 2002). Considerando la mayor conductancia térmica de las rocas más grandes, es esperable que las especies de lagartijas de mayor tamaño debieran seleccionar rocas de mayor tamaño (además de servir como mecanismo de evasión de competencia, y de obtención de terrritorio). En términos de segregación de nicho, es esperable que una misma especie, bajo distintos contextos de sintopía, muestre cambios en el uso de las rocas, mostrando una mayor amplitud de nicho en alotopía (sola) que en sintopía (junta con otra especie), lo que implicaría una disminución de la posible competencia interespecífica (al menos, en el eje del nicho tamaño de rocas).

El objetivo de este estudio es caracterizar la fauna de reptiles presentes en el Monumento Natural El Morado, comparando su abundancia relativa, la dis- 
tribución altitudinal de las distintas especies, y la preferencia y uso de rocas de distinto tamaño por las especies más abundantes, en distintos contextos de sintopía.

\section{MATERIALES Y METODOS}

\section{Area de estudio}

El Monumento Natural (MN) El Morado, de 3.000 ha de extensión, fue creado en 1974, y se encuentra ubicado en la parte superior del río Volcán, en la Cordillera de los Andes, a $90 \mathrm{~km}$. al SE de Santiago, en la Región Metropolitana de Chile (33 $30^{\text {' }}$ S, $70^{\circ} 03$ ' O; Fig. 1). El rango de altitud de esta área protegida varía entre los 1.800 y los 5.060 msnm. Las precipitaciones varían entre $1.000 \mathrm{a}$ $1.500 \mathrm{~mm} / \mathrm{año}$, las que son principalmente de carácter nival, concentradas en el invierno (Teillier $e t$ al. 1994). La vegetación de el MN El Morado se puede clasificar en cuatro grandes grupos: matorral arborescente subandino, matorral andino, estepa andina y estepa altoandina (ver revisión en Teillier et al. 1994).

\section{ABUNDANCIA RELATIVA Y DISTRIBUCIÓN ALTITUDINAL}

Entre diciembre de 2001 y marzo de 2002 se realizaron 4 salidas a terreno, las que variaron entre 2 días (16-17 enero 2002), 4 días (25-28 enero 2002) a 5 días de muestreo (20-24 diciembre 2001 y 6-10 de marzo 2002). En el área de estudio se cuantificó la presencia y abundancia relativa de reptiles mediante 20 recorridos por todo el sector. Dependiendo de las condiciones climáticas, el muestreo abarcó hasta el sector Panimávidas, Laguna Morales y Base del Glaciar San Francisco. En los recorridos ( 8 al interior de 3 cuadrantes de muestreo de 0,16 ha y 12 fuera de ellos; de longitud y tiempo variable), determinados in situ, se anotó la presencia y abundancia de las especies avistadas. El muestreo se concentró entre las 10:00 AM y 1:00 PM.

La distribución altitudinal se registró en 3 períodos de muestreo (dos en enero de 2002 y uno en marzo de 2002), en los que se contabilizó el número de ejemplares avistados en 12 recorridos de muestreo (fuera de los cuadrantes), separándolos por especie y por rango de altitud, la que fue determinada por altímetro y por GPS. Los recorridos se ubicaron de modo de abarcar todo el rango altitudinal (de alrededor de 600 metros), abarcando desde los 1.850 msnm (entrada del MN) hasta los $2.480 \mathrm{msnm}$ (base del Glaciar San Francisco). Además, los recorridos se ubicaron preferentemente en ambientes con dominancia de sustrato rocoso (microhábitat preferido por los reptiles de altura), abarcando un amplio rango de tamaños de rocas, y evitando los sectores de vegas. La abundancia y distribución altitudinal se expresó en función del número total de ejemplares contabilizados por cada piso altitudinal.

USO Y PREFERENCIA POR ROCAS DE DISTINTO TAMAÑO

En tres sectores específicos (cuadrantes 1, 2 y 3; Fig. 1), y en un total de 8 recorridos, se cuantificó la preferencia por rocas de distinto tamaño, contabilizando tanto la disponibilidad como el uso de rocas por los reptiles, separándolas en rangos de tamaño (de 10 en $10 \mathrm{~cm}$ ). La selección de los cuadrantes se realizó en base a la presencia de las distintas especies (de modo de considerar diversos contextos de sintopía), la distinta disponibilidad de sustrato rocoso así como abarcar un amplio rango de tamaños de rocas. Para el caso de la disponibilidad de rocas, los cuadrantes fueron caracterizados en su cobertura en categorías como hierba, arbusto, suelo desnudo y rocas de distintos tamaños, mediante dos transectos diagonales de $50 \mathrm{~m}$. cada uno, en los que se midió la distancia lineal cubierta por cada categoría. Para cuantificar el uso por los reptiles, se realizaron transectos semialeatorios por todo el sector, especialmente en los lugares con mayor probabilidad de encuentro (cerca de vegetación arbustiva más alta, en roqueríos). Se registró la especie y el tamaño de la roca (midiendo el diámetro mayor) donde se encontró cada individuo. Se consideraron sólo ejemplares adultos.

Finalmente, se utilizó la prueba de $\chi^{2}$ para comparar las frecuencias observadas y esperadas en cada categoría de tamaño de rocas (agrupando categorías de modo que las frecuencias esperadas fueran mayores que 1), y determinar la preferencia o evasión por cada categoría de tamaño.

Como complemento, se registró el uso de rocas de distinto tamaño por las tres especies más abundantes, en recorridos realizados en otros sectores en los que no se evaluó la disponibilidad de tamaño de rocas (y por lo tanto, en los que no se pudoestimar preferencia). 


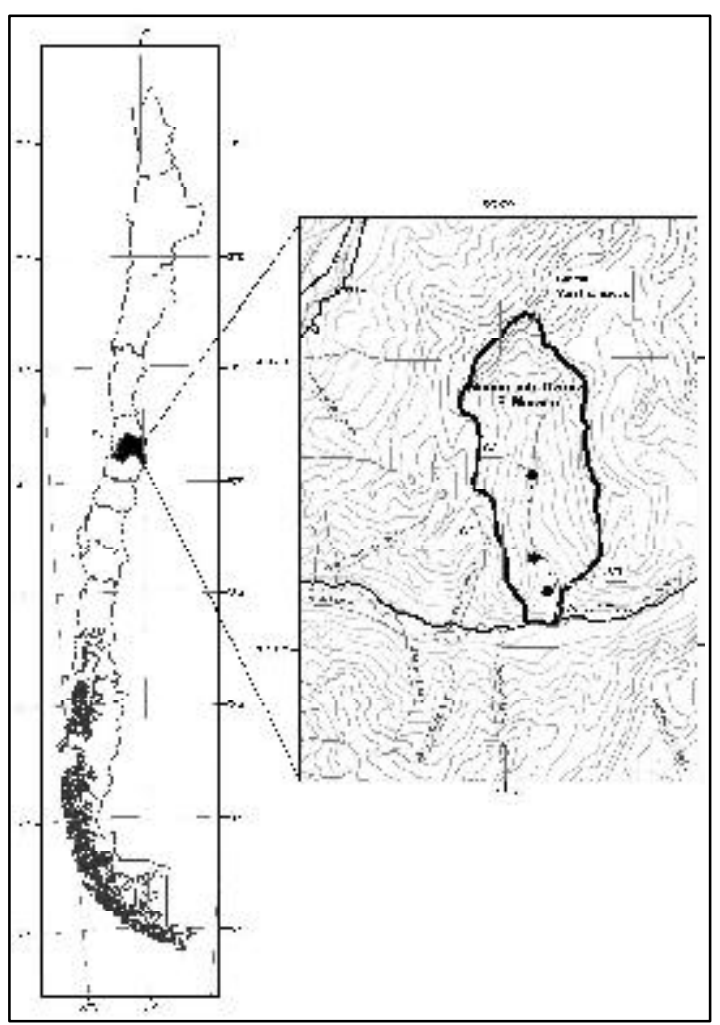

Figura 1: Localización del sector de estudio: Monumento Natural El Morado, Región Metropolitana de Chile. Los límites del área protegida se indican con línea gruesa. La línea discontinua fina indica la ubicación del sendero (donde se realizaron los recorridos de muestreo). Los círculos señalan la ubicación de los cuadrantes 1 a 3 . La línea punteada indica la ubicación aproximada del sendero (donde se realizaron los recorridos de muestreo).

FIgURE 1: Geographical location of the El Morado Natural Monument, Metropolitan Region, Chile. The thick line shows the limits of protected area. The discontinued thin line shows the track, and the circles correspond to quadrants 1 to 3 .

\section{Cuadrantes de Muestreo}

El cuadrante ubicado a menor altitud es el Cuadrante 1, a los $2.000 \mathrm{~m}$. El Cuadrante 2 se localizó a los $2.272 \mathrm{~m}$, mientras que el Cuadrante 3 se ubicó a un costado de la Laguna Morales, a 2.400 m (Fig. 1). Las especies arbustivas dominantes fueronAdesmia gracilis, Chuquiraga oppositifolia, Berberis empetrifolia, y las herbáceas perennes dominantes fueron Acaena sp. y Senecio sp. El microhábitat rocoso es el dominante en los tres sectores, con valores de $93,8 \%$ (Cuadrante 3), 53,5\% (Cuadrante 1) y $49,1 \%$ (Cuadrante 2), seguido por el estrato arbusti- vo, con valores de 18,8\% (Cuadrante 2), 8,9\% (Cuadrante 1) y $4,4 \%$ (Cuadrante 3 ).

\section{RESULTADOS}

\section{Abundancia Relativa DE REPTILES}

Se encontraron seis especies de reptiles, todas endémicas de Chile: cuatro lagartos del géneroLiolaemus: la lagartija parda de El Morado (L. altissimus moradoensis, de aquí en adelante L. moradoensis, aceptando la propuesta de Pincheira-Donoso \& Núñez (2005)), el lagarto nítido (L. nitidus), la lagartija negroverdosa (L. nigroviridis) y el lagarto de Lo Valdés (L. valdesianus). Las otras dos especies registradas son las culebras de cola larga (Philodryas chamissonis) y de cola corta (Tachymenis chilensis). En el total de 4 períodos, se contabilizaron 257 observaciones de las seis especies. La especie más abundante fue Liolaemus moradoensis, con 105 registros, lo que corresponde al $40,9 \%$, seguido por $L$. nigroviridis, con 97 avistamientos (37,7\%). La tercera especie más abundante correspondió a $L$. valdesianus, con 44 registros $(17,1 \%)$. Las especies menos abundantes fueron L. nitidus $(\mathrm{n}=7 ; 2,7 \%)$, Tachymenis chilensis $(\mathrm{n}=3 ; 1,2 \%)$ y Philodryas chamissonis, con una sola observación.

\section{DistRIBUCIÓN ALTITUDINAL}

A pesar que el rango de altitud estudiado no fue muy amplio (alrededor de $600 \mathrm{~m}$ ), las distintas especies muestran distintos patrones de distribución altitudinal. Así, L. nitidus fue registrado sólo en el sector de pircas cercano a la administración de CONAF, a una altitud menor a los $1.900 \mathrm{~m}$ (Fig. 2). En el otro extremo altitudinal, L. moradoensis y $L$. valdesianus se encuentran a altitudes mayores a $2.150 \mathrm{~m}$; siendoL. moradoensis la especie más abundante en el rango de mayor altitud, con cerca del $90 \%$ de abundancia a alturas mayores a $2.350 \mathrm{~m}$ (Fig. 2). La especie que ocupó un mayor rango de altitud fue $L$. nigroviridis, presente entre $\operatorname{los} 1.850$ a los 2.350 , y con un máximo de abundancia relativa a los $2.100 \mathrm{~m}$ (Fig. 2). A pesar del bajo número de ejemplares registrados, la culebra de cola corta se encontró entre 1.850 y los $2.250 \mathrm{~m}$ (Fig. 2). Agrupando todas las especies, la mayor abundancia absoluta de reptiles se encuentra sobre los $2.150 \mathrm{~m}$. 


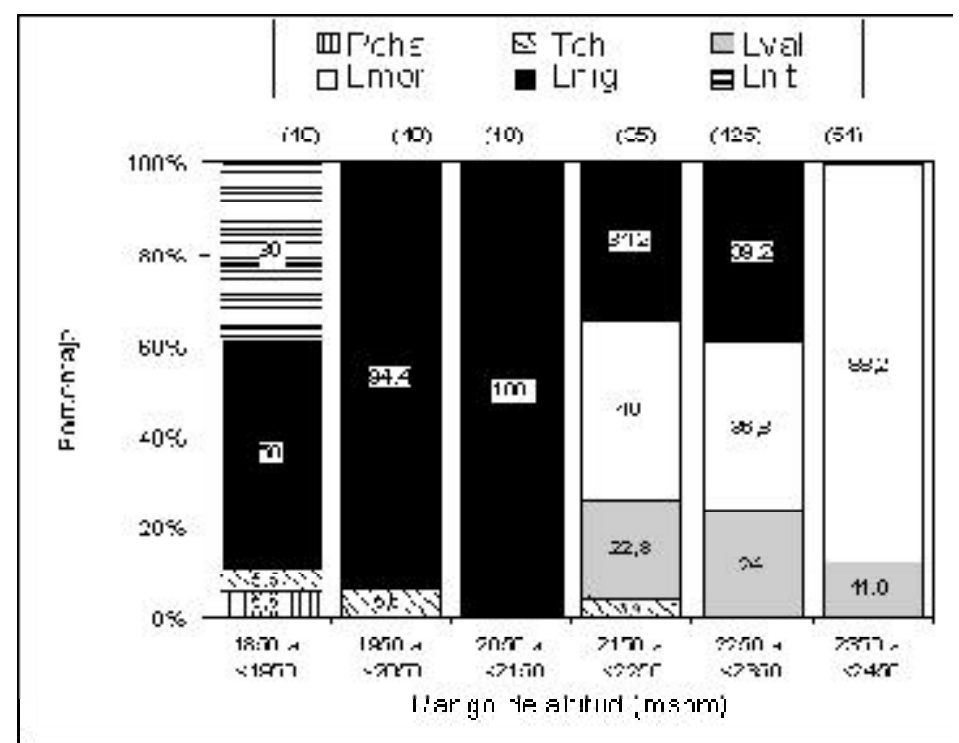

FIgURA 2: Distribución altitudinal de reptiles en el Monumento Natural El Morado, Chile central. Para cada especie, el número al interior de cada barra indica el porcentaje de abundancia en cada rango de altitud. (Abreviaturas de las especies: $\mathrm{Pcha}=$ Phylodryas chamissonis $;$ Tchi $=$ Tachymenis chilensis $;$ Lval $=$ Liolaemus valdesianus $;$ Lmor $=L$. moradoensis $;$ Lnig $=$ L. nigroviridis $;$ Lnit $=L$. nitidus $)$. Entre paréntesis se indica el total de registros en cada rango de altitud.

FIGURE 2: Altitudinal distribution of lizards and snakes at El Morado Natural Monument, central Chile. For each species, the number inside the bars shows the percentage of abundance in each altitudinal range. Abreviations of species: Pcha = Phylodryas chamissonis $;$ Tchi $=$ Tachymenis chilensis $;$ Lval = Liolaemus valdesianus $;$ Lmor = L. moradoensis $;$ Lnig = L. nigroviridis; Lnit $=$ L. nitidus). The total of records in each altitudinal range is give in parentheses.

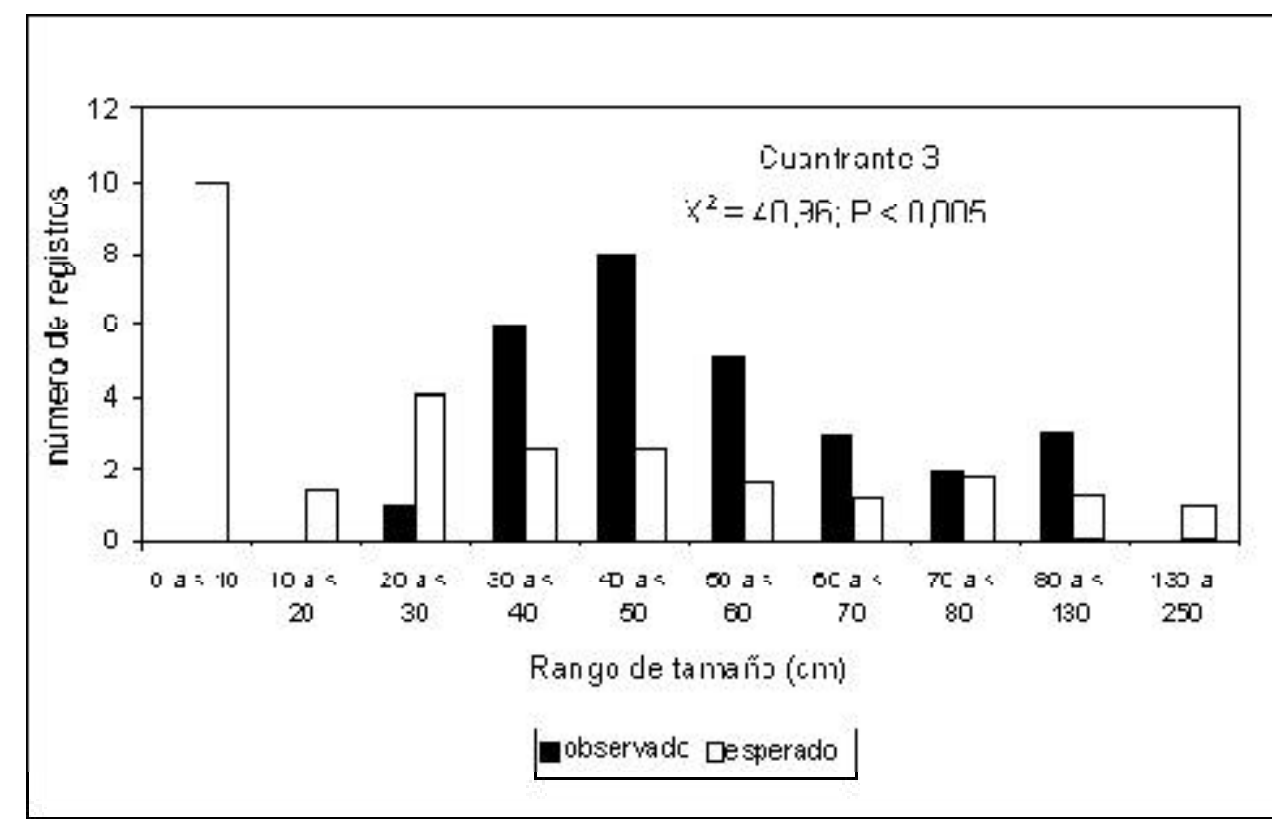

Figura 3: Preferencia por tamaño de rocas en Liolaemus moradoensis, Monumento Natural El Morado (n= 28; g.1. = 9).

Figure 3: Boulder size preference in Liolaemus moradoensis, El Morado Natural Monument (n=28; d. f. = 9). 
Reptiles en el Monumento Natural El Morado: Melda, J.

USO Y PREFERENCIA POR ROCAS DE DISTINTO TAMAÑO

Liolaemus moradoensis: Esta especie fue estudiada en el Cuadrante 3, sector en que mostró una preferencia significativa por rocas entre 30 a $60 \mathrm{~cm}\left(\chi^{2}=\right.$ 40,96; $\mathrm{P}<0,005$; g.l. $=9 ; \mathrm{n}=28$ individuos registrados), a la vez que evadió las rocas menores a $30 \mathrm{~cm}$ y mayores a $130 \mathrm{~cm}$ (Fig. 3).

Liolaemus nigroviridis: Esta especie fue estudiada en el Cuadrante 1 (donde fue la única especie registrada) y en el Cuadrante 2 (en sintopía con $L$. valdesianus). En el Cuadrante 1, L. nigroviridis prefirió significativamente las rocas entre 30 a $120 \mathrm{~cm}$, evadiendo las rocas menores a $30 \mathrm{~cm}$ y las mayores a $2 \mathrm{~m}$ $\left(\chi^{2}=38,95 ; \mathrm{P}<0,005 ;\right.$ g.l. $=8 ; \mathrm{n}=16$, Fig. 4.a). En el Cuadrante 2, esta especie prefirió las rocas entre 30 a $60 \mathrm{~cm}$, evadiendo las rocas menores a $30 \mathrm{~cm}\left(\chi^{2}=\right.$ 10,81; $\mathrm{P}<0,05 ;$ g.1. = 4; $\mathrm{n}=10$, Fig. 4.b).

Liolaemus valdesianus: En el Cuadrante 2, y a pesar del bajo número de ejemplares registrados, esta especie mostró una preferencia por las rocas mayores a $90 \mathrm{~cm}\left(\chi^{2}=12,18 ; \mathrm{P}<0,005 ;\right.$ g.l. $=2 ; \mathrm{n}=$ 7, Fig. 5).
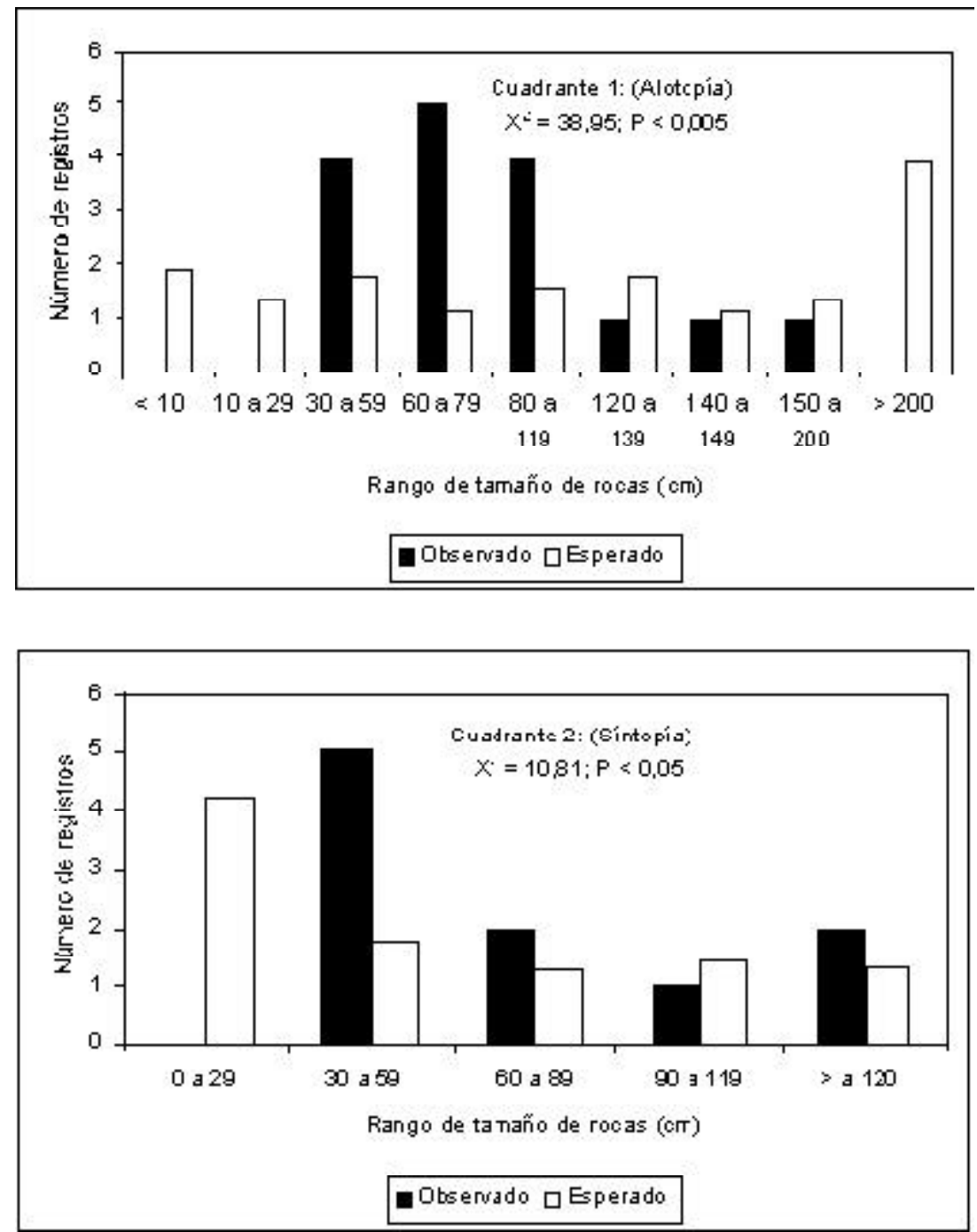

Figura 4: Preferencia por tamaño de rocas en Liolaemus nigroviridis (Monumento Natural El Morado), en alotopía (arriba; Cuadrante 1: $\mathrm{n}=16$; g.l. = 8) y en sintopía con L. valdesianus (abajo; Cuadrante 2: $\mathrm{n}=10$; g. 1. = 4).

FIGURE 4: Boulder size preference in Liolaemus nigroviridis, El Morado Natural Monument, in allotopy (top; quadrant $1 ; \mathrm{n}=16$; d. f. $=8$ ), and in sintopy with L. valdesianus (bottom; Quadrant $2 ; \mathrm{n}=10 ;$ d. f. $=4$ ) . 


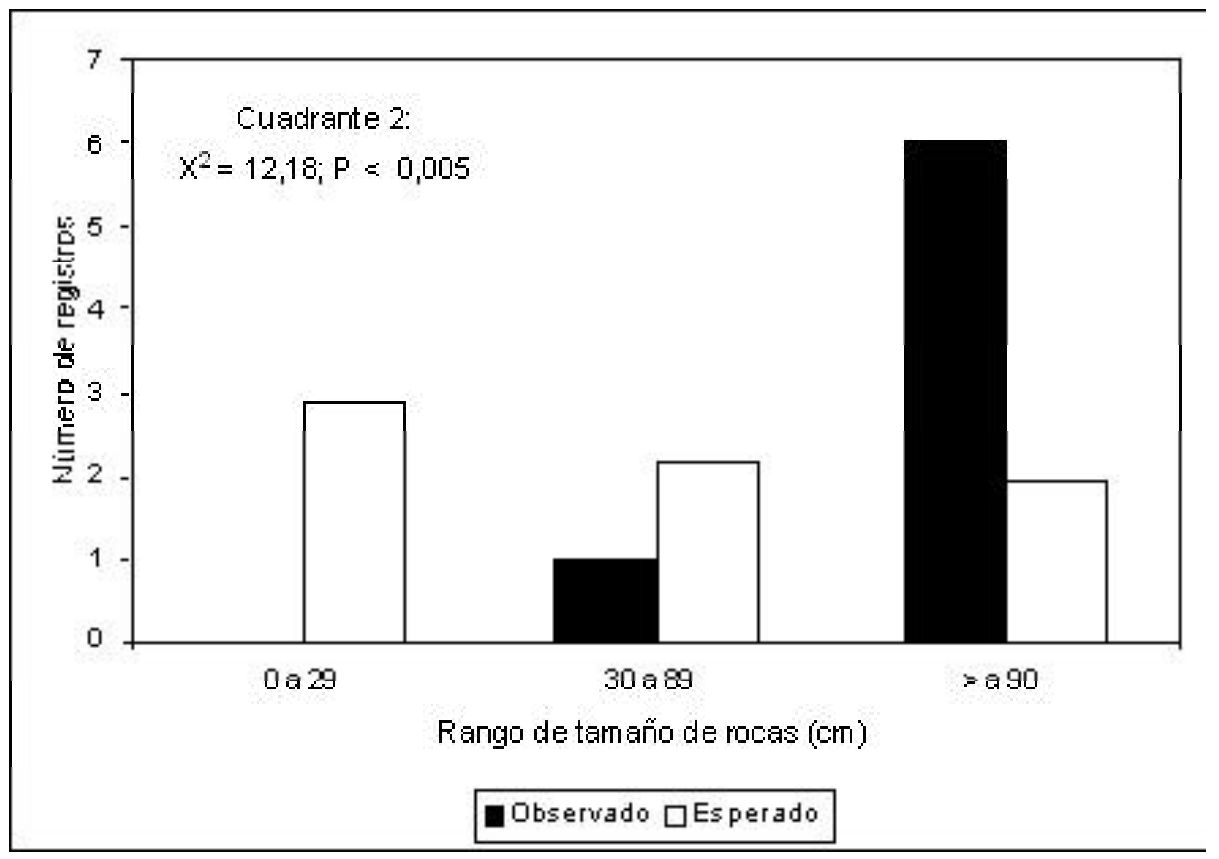

FIgURA 5: Preferencia por tamaño de rocas en Liolaemus valdesianus, Monumento Natural El Morado (n = 7; g.l. = 2).

FIGURE 5: Boulder size preference in Liolaemus valdesianus, El Morado Natural Monument ( $\mathrm{n}=7$; d. f. = 2).

Los resultados de preferencia fueron complementados, para las tres especies, con el registro del uso de rocas en otros sectores, en los que no se evaluó disponibilidad de rocas (por lo que no se puede determinar preferencia o evasión significativa). $L$ moradoensis usó una mayor proporción de rocas entre 20 a $80 \mathrm{~cm}(\mathrm{n}=42)$, mientras que $L$. nigroviridis fue registrado en mayor porcentaje en rocas entre 30 a $110 \mathrm{~cm}(\mathrm{n}=37$; ANEXO I). En contraste a las anteriores especies y en forma similar a lo registrado en el Cuadrante $3, L$. valdesianus se registró más frecuentemente en rocas mayores a 130 cm ( $n=29$; ANEXO I). En general, estas observaciones de uso de rocas son coincidentes con los de preferencia por rocas de distinto tamaño.

\section{DISCUSION}

El registro de 6 especies de reptiles en el Monumento Natural El Morado (Región Metropolitana, Chile) complementa lo documentado anteriormente, donde se habían registrado sólo 3 especies $(L$. moradoensis, L. valdesianus y Tachymenis chilensis,
CONAF 1988); por lo tanto, las especies $L$. nigroviridis, L. nitidus y Philodryas chamissonis constituyen nuevos registros para esta área protegida. En relación a L. moradoensis, antes considerada como subespecie de L. altissimus y recientemente elevada a especie plena (Pincheira-Donoso \& Núñez 2005), es importante destacar que tiene su localidad tipo en El Morado (Donoso-Barros 1966).

En cuanto a la abundancia relativa, las especies más abundantes fueron L moradoensis y L. nigroviridis, representando más del $79 \%$ de abundancia de reptiles. En este sentido, son escasos los registros de abundancia cuantificada de reptiles (ver revisión en Mella 2005a).

A pesar que el rango de altitud analizado no fue amplio $(600 \mathrm{~m})$, se observaron patrones de distribución altitudinal diferentes entre las especies. Liolaemus nitidus se encontró sólo en el sector más bajo, por debajo de los $1.900 \mathrm{msnm}$, valor inferior (aunque dentro del rango) a los máximos registrados en otros estudios (2.300: Veloso \& Navarro 1988; 2.400: Carothers et al. 1998; 2.200: Díaz \& Simonetti 1997; 3.050: Mella 2005a). La ausencia de registros en sectores más altos pudiera deberse a 
la discontinuidad de ambientes con rocas de gran tamaño (utilizadas más frecuentemente por esta especie), muy escasas en el tramo intermedio del rango altitudinal estudiado. Liolaemus nigroviridis mostró el mayor rango de altitud (1.850 a los 2.350 msnm), concordando con el rango registrado en otros estudios (1.600 - 2.000: Veloso \& Navarro, 1988; 1.700 - 3.000: Carothers et al., 1998; 1.500 2.400: Díaz \& Simonetti 1997; 500 - 3.370: Mella 2005a). Por otra parte, L. valdesianus se encontró entre los 2.150 a los $2.450 \mathrm{msnm}$, ampliando el rango superior conocido, ya que Veloso \& Navarro (1988) indican un rango entre 1.850 a $2.200 \mathrm{msnm}$. Finalmente, L. moradoensis se ubicó entre los 2.150 a los $2.450 \mathrm{msnm}$, concordando con su distribución conocida, entre 1.700 a 3.500 msnm (Mella 2005a, como L. altissimus moradoensis).

Entre los factores que se han postulado para explicar los patrones de distribución altitudinal de reptiles se han mencionado las asociaciones con la vegetación (Zunino \& Riveros 1981), restricciones termoregulatorias (Carothers et al. 1998) y masa corporal (Carothers et al. 1997). En el caso del MN EL Morado, la presencia y abundancia de reptiles (todos saxícolas) se relaciona directamente con la disponibilidad de microhábitats rocosos. Así, la baja presencia de ejemplares entre los 1.900 y 2.100 msnm puede explicarse, al menos en parte, por la baja proporción de ambientes rocosos (con dominancia de arbustos, hierbas $(35,7 \%$ en Cuadrante 1) y piedras pequeñas), mientras que la alta abundancia de lagartijas entre los 2.200 a los 2.450 coincide con la dominancia de alta cobertura rocosa (93,8\% en Cuadrante 3 ), y en particular, con rocas de mayor tamaño.

En cuanto a la preferencia por rocas de distinto tamaño, L. moradoensis prefirió las rocas entre 30 a $60 \mathrm{~cm}$ de longitud, mientras que $L$. nigroviridis en alotopía mostró una preferencia más amplia, por rocas entre 30 a $120 \mathrm{~cm}$. En contraste a las dos especies anteriores, L. valdesianus prefirió las rocas de mayor tamaño, sobre $90 \mathrm{~cm}$. Lo anterior estaría explicado en parte por el tamaño de las especies (lo que se relaciona directamente tanto con el tamaño del territorio ocupado como con la inercia térmica): para L. moradoensis, el tamaño máximo es de $170 \mathrm{~mm}$ (Longitud total), similar a L. nigroviridis (166 mm), tamaños menores a L. valdesianus, con un máximo de $228 \mathrm{~mm}$ (Donoso-Barros 1966; Mella 2005a; sin considerar colas regeneradas). Con- cordando con lo esperado, las especies más pequeñas prefieren rocas de menor tamaño (y similares entre sí) y la especie más grande prefiere las rocas de mayor tamaño. Diversos factores pueden explicar este resultado, como aspectos termoregulatorios, evasión de la competencia interespecífica, selección de tamaño de territorio y altura de percha, entre otros. Considerando aspectos termorregulatorios, la selección de rocas grandes (con mayor inercia térmica que las rocas pequeñas) favorecería a la especie de mayor tamaño (L. valdesianus), la que puede ampliar su periodo de actividad (por tigmotermia; Carothers et al. 1998). Además, el poseer rocas de mayor tamaño puede implicar tener un territorio más óptimo, con el consecuente aumento de recursos alimenticios y de favorecimiento en la elección de pareja y en la formación de harenes. De acuerdo a Carothers et al. (2001), la selección de rocas grandes por L. leopardinus y de rocas pequeñas por $L$. monticola estaría explicando incluso sus límites distribucionales, mientras que la selección de rocas grandes por L. kuhlmanni estaría explicado básicamente por evasión de depredación, ya que esta especie es territorial y de coloración no críptica, evitando las zonas de arenales (Fuentes \& Cancino 1979). La relación entre selección de rocas de diferente tamaño y el tamaño del ámbito de hogar y la formación de harenes ha sido documentada para Phymaturus flagellifer por Habit \& Ortiz (1994), quienes encontraron que en áreas con rocas grandes y numerosas grietas, los machos adultos tenían ámbitos de hogar relativamente pequeños (solapados con los de las hembras), y tienden a ser territoriales y con harenes, mientras que en áreas con dominancia de rocas pequeñas y pocas grietas, los ámbitos de hogar de los machos adultos eran mayores (y similares a los de las hembras).

Carothers et al. (1998) estudiando diez especies de lagartijas de Chile central (dentro de las cuales se encuentra L. nigroviridis), encontraron que el uso de rocas es mayor en el atardecer que en la mañana. En este estudio no se registraron las especies al atardecer, pero es esperable que la preferencia por distintos tamaños de roca sea más evidente en el atardecer en otros sectores, en los que se encuentren reptiles con actividad bimodal más acentuada. Al contrario, en El Morado (una microcuenca muy cerrada), es esperable que la actividad de los reptiles se concentre principalmente en la mañana.

Por otra parte, Carothers et al. (1997) mostraron 
diferencias térmicas en adultos y juveniles de varias especies de Liolaemus, las que podrían explicar distintas conductas de uso de microhábitats. En este estudio, el uso y preferencia de reptiles por rocas se centró exclusivamente en los adultos, por lo que se minimiza el efecto que podrían producir el incluir ejemplares juveniles (los juveniles de L. valdesianus podrían sobreponerse en su preferencia de tamaño de rocas con los adultos de las otras especies más pequeñas, con lo que se ocultaría la segregación observada en distintos contextos de sintopía).

Los resultados muestran finalmente un patrón significativo en términos de uso y desplazamiento de nicho. Para el eje del nicho tamaño de rocas $L$. nigroviridis se sobrepone a $L$. moradoensis, el cual tiene un nicho más estrecho; mientras que $L$. valdesianus tiene un nicho no sobrepuesto, centrándose en un mayor tamaño de rocas. Es interesante destacar un desplazamiento del nicho en $L$. nigroviridis, la única especie que se estudió tanto en alotopía (sola, en el Cuadrante 1) como en sintopía (junto con L. valdesianus, en el Cuadrante 2). En efecto, L. nigroviridis utiliza un rango más amplio de rocas en alotopía (30 a $120 \mathrm{~cm}$ ) que en sintopía $(30$ a $60 \mathrm{~cm})$, posiblemente para minimizar el efecto de la competencia por uso de rocas con $L$. valdesianus (de mayor tamaño, supuestamente dominante), que utiliza preferentemente las rocas de mayor tamaño. Un patrón similar de desplazamiento de nicho (aunque en otro eje del nicho: altura de la percha en microhábitat arbóreo) fue registrado por Medel et al. (1988) para L. tenuis en el sur de Chile. Esta lagartija arborícola, en alopatría se ubica preferentemente en la base de los árboles, a alturas menores de $30 \mathrm{~cm}$, mientras que en simpatría con $L$. pictus, es desplazada y se ubica entre los 30 a los 60 cm. L. pictus, especie también arborícola y aparentemente dominante en términos competitivos, se ubica en la porción inferior de los troncos, a alturas menores de $30 \mathrm{~cm}$ (Medel et al. 1988).

Los antecedentes aportados por este estudio son útiles desde varias perspectivas. Por una parte, permiten ampliar los registros de presencia, abundancia relativa y distribución altitudinal de las lagartijas en un ambiente particular, antecedentes básicos para evaluar la representatividad y estado de conservación de las especies (sobre todo en un área protegida). Por otra parte, los resultados sobre preferencia por distintos tamaños de roca y el desplazamiento de nicho encontrado bajo distintos contextos de sintopía, son valiosos como ejemplos para apoyar teorías ecológicas (como la de nicho), a la vez que abren nuevas interrogantes para estudios más detallados de las preferencias por sustratos rocosos de éstas y otras lagartijas saxícolas. Por ejemplo: la distribución altitudinal de L. nitidus en este estudio (restringido sólo a los sectores más bajos) pudiera relacionarse con posible competencia por uso de rocas con L. valdesianus (especie de tamaño corporal similar), presente en los sectores más altos. Aproximaciones experimentales debieran utilizarse para cuantificar en mayor detalle el uso y preferencia de hábitats rocosos por las lagartijas, y sus relaciones en términos de nicho.

\section{AGRADECIMIENTOS}

A la Corporación Nacional Forestal (CONAF) por brindar las facilidades de acceso al área protegida, y en especial al administrador y guardaparques de El Morado. A Andrea Urquiza por su asistencia en terreno. A Fabrizio Frugone, quien gentilmente elaboró la Figura 1. Cuatro revisores anónimos ayudaron a mejorar notoriamente el texto. Este estudio fue financiado por el Proyecto de Investigación Interno $N^{\circ} 10$, del año 2001, de la Universidad Central de Chile.

\section{BIBLIOGRAFIA}

Carothers, J., S.F. Fox, P.A. Marquet \& F.M. Jaksic. 1997. Thermal characteristics of ten Andean lizards of the genus Liolaemus in central Chile. Revista Chilena de Historia Natural 70: 297-309.

Carothers, J., P.A. Marquet \& F.M. Jaksic. 1998. Thermal ecology of a Liolaemus lizard assemblage along an Andean altitudinal gradient in Chile. Revista Chilena de Historia Natural 71:39-50

Carothers, J., F.M. Jaksic \& P.A. Marquet. 2001 Altitudinal zonation among lizards of the genus Liolaemus: questions answered and unanswered questions. Revista Chilena de Historia Natural 74: 313-316.

CONAF, 1988. Plan de Manejo Monumento Natural El Morado. Unidad de Gestion Patrimonio Silvestre. Documento de trabajo $\mathrm{N}^{\circ} 256,98 \mathrm{pp}$.

Díaz, I. \& J. Simonetri. 1997. Vertebrados en Areas Silvestres Protegidas: Reptiles de la Reserva Nacional Río Clarillo, Chile central. Vida Silvestre Neotropical 5(2):140-142.

Donoso-Barros, R. 1966. Reptiles de Chile. Ediciones 
de la Universidad de Chile, Santiago.

Fuentes, E.R. \& J. Cancino. 1979. Rock-ground patchiness in a simple Liolaemus lizard community. Journal of Herpetology 13:343-350.

Habit, H. \& J.C. Ortiz. 1994. Ambito de hogar de Phymaturus flagellifer (Reptilia: Tropiduridae). Boletín de la Sociedad de Biología de Concepción (Chile) 65: 149-152.

JAKSIC, F.M. 1996. ECOLOGIA DE LOS VERTEBRADOS DE CHILE. Ediciones Universidad Católica de Chile, Santiago, 262 pp.

JAKSIC, F. M. \& H. NúÑEZ. 1979. Escaping behavior and morphological correlates in two Liolaemus species of central Chile (Lacertilia: Iguanidae). Oecologia 42: 119-122.

Jerez, V. \& J.C. Ortiz. 1975. Distribución altitudinal del género Liolaemus (Squamata-Iguanidae) en el Cerro La Campana (Parque Nacional La Campana). Anales del Museo de Historia Natural de Valparaíso 8:58-60.

M arquet, P.A., J.C. Ortiz, F. Bozinovic \& F.M. Jaksic. 1989. Ecological aspects of thermoregulation at high altitudes: the case of Andean Liolaemus lizards in northern Chile. Oecologia 81: 16-20.

Medel, R.G., P.A. Marquet \& F.M. Jaksic. 1988. Microhabitat shifts of lizards under different contexts of sympatry; a case study with South American Liolaemus. Oecologia 76:567-569.

Mella, J. 2002. Dieta del cernícalo (Falco sparverius) y del Tucúquere (Bubo magellanicus) en un ambiente cordillerano de Chile central. Boletín Chileno de Ornitología 9:34-37.

Mella, J. 2005a. Guía de campo de reptiles de Chile: Zona central. Peñaloza APG, F Novoa \& M. Contreras (Eds.). Ediciones del Centro de Ecología Aplicada Ltda. 147 pp + xii.

Mella, J. 2006a. Dinámica Poblacional de Bufo spinulosus (Anura: Bufonidae) en el Monumento Natural El Morado (Región Metropolitana). Noticiario Mensual Museo Nacional de Historia Natural, Chile, № 357: 19-22.

Mella, J. 2006b. Micromamíferos en el Monumento Natural El Morado: abundancia relativa y cambios estacionales. Noticiario Mensual Museo Nacional de Historia Natural, Chile, $\mathrm{N}^{\circ} 357$ : 10-18.

Mella, J. 2006c. Cambios estacionales en la avifauna del Monumento Natural El Morado, Cordillera de Santiago. Boletín Chileno de Ornitología 11: $2-10$.

Moreno, R., J. Moreno, F. Torres-Pérez \& J.C. Ortiz. 2000. Reptiles del Parque Nacional "Nevado de Tres Cruces" (III Región, Chile). Boletín de la Sociedad de Biología de Concepción, Chile 71: 41-43.

NúÑEZ, H. 1996. Autoecología comparada de dos especies de lagartijas de Chile central. Publicación Ocasional del Museo Nacional de Historia Natural, Chile 50:5-60.

Ortiz, J.C. 1974. Reptiles del Parque Nacional Vicente Pérez Rosales. Anales del Museo de Historia Natural de Valparaíso 7: 317-326.

ORTIZ J.C \& H. IBARRA-Vidal. 2005. Anfibios y reptiles de la Cordillera de Nahuelbuta. Pp. 427-440, en: Smith-Ramírez C, JJ Armesto \& C Valdovinos (eds.), Historia, Biodiversidad y Ecología de los Bosques Costeros de Chile. Editorial Universitaria, Santiago, Chile. 708 pp.

PiAnKa E.R. 1973. The structure of lizard communities. Annual Review of Ecology and Systematics 4: 53-74.

Schoener T.W. 1974. Resource partitioning in ecological communities. Science 185: 27-39.

Teillier, S. 2003. Flora del Monumento Natural El Morado: Addenda et corrigenda. Gayana Botánica 20(2):94-100.

Teillier, S., A. Hoffmann, F. SaAvedra \& L. Pauchard. 1994. Flora del Parque Nacional El Morado (Región Metropolitana, Chile). Gayana Botánica 51:13-47.

Veloso, A. \& J. Navarro. 1988. Lista sistemática y distribución geográfica de anfibios y reptiles de Chile. Bolletino del Museo Regionale di Scienze Naturali 6: 481-539.

Vidal, M., J.C. Ortiz \& A. Labra. 2002. Sexual and age differences in ecological variables of the lizard Microlophus atacamensis (Tropiduridae) from Northern Chile. Revista Chilena de Historia Natural 75: 283-292.

Zunino, S. \& G. Riveros. 1981. Distribución de reptiles y su relación con la vegetación en "Parque Nacional La Campana"' Chile central. Anales del Museo de Historia Natural de Valparaíso 14:185-188. 
Gayana 71(1), 2007

Anexo I. Uso de tamaño de rocas en tres especies de Liolaemus, en sectores en que no se evaluó disponibilidad (de tamaño de rocas). Monumento Natural El Morado, Región Metropolitana.

ANNEx I. Use of size of rocks in three species of Liolaemus, in sectors where the availability (in sizes of rocks) has not been evaluated. El Morado Natural Monument, Metropolitan Region, central Chile.

\begin{tabular}{lccc}
\hline RANGO DE TAMAÑo & \multicolumn{3}{c}{ EsPeCIE } \\
DE ROCA (CM) & L. nigroviridis & L. moradoensis & L. valdesianus \\
\hline & 0 & 0 & 0 \\
10 & 0 & 1 & 0 \\
10 a 19 & 1 & 8 & 0 \\
20 a 29 & 5 & 8 & 0 \\
30 a 39 & 5 & 1 & 0 \\
40 a 49 & 9 & 7 & 2 \\
50 a 59 & 2 & 4 & 1 \\
60 a 69 & 3 & 8 & 1 \\
70 a 79 & 3 & 0 & 3 \\
80 a 89 & 4 & 0 & 3 \\
90 a 99 & 3 & 1 & 0 \\
100 a 109 & 1 & 2 & 1 \\
110 a 119 & 0 & 1 & 2 \\
120 a 129 & 1 & 1 & 16 \\
130 a 320 & $\mathbf{3 7}$ & $\mathbf{4 2}$ & $\mathbf{2 9}$ \\
\hline Total & & & \\
\hline
\end{tabular}

Fecha de recepción: 26.04.06

Fecha de aceptación: 15.03.07 